\title{
FALSOS AMIGOS LÉXICOS Y RECURSOS LEXICOGRÁFICOS ELECTRÓNICOS EN EL ÁMBITO ITALOESPAÑOL
}

\author{
ROSA MARTÍN GASCUEÑA \\ Universidad Complutense de Madrid, España \\ MARÍA TERESA SANMARCO BANDE \\ Universidad de Santiago de Compostela, España
}

\begin{abstract}
Resumen. La lexicografía contrastiva dedicada al par de lenguas italianoespañol ha experimentado una notable evolución en el último decenio; pero sigue habiendo campos en los que la información proporcionada en los diccionarios todavía es insuficiente. Sucede a menudo con los falsos amigos léxicos (términos del castellano como defraudar, larva, tesitura o del italiano, indiano, salire, versare), debido a la engañosa proximidad entre ambos idiomas. Frecuentemente, esta sensación de cercanía impide reconocer las diferencias existentes, reforzando el peso de los estereotipos culturales e invisibilizando así los elementos distintivos de cada una de las dos lenguas y de las dos culturas. La finalidad de nuestro estudio es mostrar cómo se refleja esta divergencia lingüístico-cultural en los diccionarios bilingües italoespañoles más utilizados y, para ello, nos centraremos en el tratamiento de los falsos amigos léxicos presentes en las obras más recientes, correspondientes a las editoriales Garzanti y Zanichelli. Después de mostrar cómo se refleja esta información en los diccionarios en uso, ofrecemos una serie de recursos electrónicos (diccionarios, bases de datos, aplicaciones... algunos en proceso de elaboración, como MultiGenera y MultiComb), exponiendo cómo pueden complementar los contenidos sobre los falsos amigos léxicos. Nuestro objetivo final es ofrecer una panorámica de los recursos lexicográficos disponibles para perfilar las definiciones de los falsos amigos, contribuyendo además a la crítica metalexicográfica.
\end{abstract}

Palabras clave: falsos amigos léxicos, italiano-español, lexicografía bilingüe, recursos lexicográficos electrónicos, MultiGenera, MultiComb

\section{INTRODUCCIÓN}

Los diccionarios han experimentado un cambio notable a lo largo de la última década, con el fin de adaptarse a una realidad compleja en la que la ingeniería lingüística y la metalexicografía juegan un papel fundamental. No solo han cambiado el modo de elaborar diccionarios, la tipología de las obras, la variedad 
de formatos de presentación, la naturaleza de las consultas de los usuarios, incluso la sociedad para la que estas obras lexicográficas han sido escritas (Gouws, 2017: 20). A la par, como consecuencia de todo ello y también gracias al impulso de los estudios teóricos en lexicografía, la arquitectura del propio artículo, el núcleo de toda obra lexicográfica, ha incorporado contenidos referidos a aspectos semánticos, sintácticos, culturales, pragmáticos o incluso propios de la comunicación oral (Sanmarco Bande, 2018: 118-119), dando lugar a obras más complejas y completas.

Con todo, siguen existiendo parcelas de información pendientes de ser incluidas o que, al menos, deberían ser tratadas de forma homogénea dentro el artículo lexicográfico. En el ámbito de las lenguas afines, para las que hemos tomado como ejemplo el par de lenguas castellano-italiano, esto sucede, por ejemplo, con los falsos amigos, las palabras culturales que carecen de equivalente en la lengua de llegada o las expresiones propias del lenguaje oral.

En este estudio nos centraremos en el tratamiento lexicográfico de los falsos amigos léxicos, interferencias lingüísticas a menudo difíciles de resolver y a las que todos los diccionarios bilingües deberían prestar especial atención, para facilitar la prevención de errores en el aprendizaje de una lengua afín.

Observaremos qué información específica se ha recogido en las obras más utilizadas y recientes en ámbito italoespañol, mostrando ejemplos y extrayendo conclusiones con respecto al tratamiento lexicográfico de los falsos amigos léxicos. Y a continuación enumeraremos otros recursos lexicográficos, entre los que se encuentran MultiGenera y MultiComb (dos novedosos proyectos de generación automática de frases), como herramientas eficaces para completar los contenidos referidos a estos términos engañosos. De este modo, proporcionaremos una batería de recursos electrónicos que, usados con fines lexicográficos, constituyen una eficaz ayuda en la descripción de los falsos amigos léxicos entre lenguas afines.

\section{DIDÁCTICA DE LAS LENGUAS AFINES}

El español y el italiano son dos lenguas próximas, cuyas culturas también cuentan con numerosas semejanzas. La interacción es un importante dinamizador del aprendizaje y precisamente entre lenguas afines se crea una interlengua eficaz, que aprovecha recursos propios de la lengua materna, acelerando así los tiempos de aprendizaje.

Como contrapartida, en los primeros niveles de aprendizaje, dicha interlengua tiende a la asimilación y a la banalización de las características intrínsecas de la segunda lengua (a partir de ahora, L2). Esta sensación de familiaridad entre ambos idiomas impide identificar los contenidos divergentes, invisibilizando los elementos distintivos de cada una de las dos lenguas y de las dos culturas (Calvi, 1995: 94).

Por esta razón, los docentes de lenguas afines deben prestar especial atención a favorecer la creación de una interlengua correcta y dinámica, estableciendo 
modalidades didácticas que ayuden a controlar este proceso, en el que las obras lexicográficas deben jugar un papel primordial, como fuentes de consulta y verificación.

Con el objeto de ejemplificar esta afirmación, hemos escogido el campo de los falsos amigos léxicos, puesto que constituye uno de los escollos fundamentales en el aprendizaje de una lengua afín; de tal suerte que la diccionarística contemporánea ha comenzado a tratar estos contenidos en la microestructura de las obras lexicográficas.

\section{FALSOS AMIGOS LÉXICOS}

El léxico de lenguas afines se puede clasificar en tres grupos (Calvi, 1995: 87-90): aquel que muestra una sinonimia y equivalencia casi absoluta entre significante y significado (pared: parete, practicar: praticare), el léxico que difiere completamente de una lengua a otra (hai stracciato la tenda con delle forbici: has rasgado la cortina con unas tijeras) y, por último, aquel que presenta una equivalencia formal (homonimia) o una gran semejanza (paronimia), con diferencias de significado (falsos amigos, como burro: mantequilla o medicare: tratar una herida).

Podemos definir el falso amigo como la interferencia lingüística entre dos lenguas, generada por una semejanza formal de términos o expresiones cuyo significado es total o parcialmente diferente. Varios autores se han ocupado de teorizar y clasificar la tipología de los falsos amigos: entre los más relevantes citaremos a Álvarez Lugrís (1997: 83), Mello y Satta (1998: 12-13) o Carlucci y Díaz Ferrero (2007: 177-183). Si nos centramos en la tipología específica de los falsos amigos léxicos, podemos observar cómo se establecen dos clases de términos: los falsos amigos totales, cuyo contenido semántico carece de relación (con ejemplos como idiosincrasia: aversión, disgusto: asco) o los falsos amigos parciales, que comparten parcelas de contenido semántico (buona notte: buenas noches, me voy a dormir, fomentare: potenciar algo negativo).

Si es primordial identificar los términos cuya igualdad en la forma no se corresponde luego en el contenido, no es menos importante acotar el significado de los falsos amigos parciales. De ello se ocupa la didáctica de la L2, la lingüística contrastiva y la lexicografía bilingüe, tratando de prevenir al usuario, proporcionándole la información necesaria para mantener una interlengua correcta.

\section{SOPORTE LEXICOGRÁFICO}

El estudio de esta interferencia lingüística se ha extendido también a la lexicografía teórica y práctica, de modo que tanto los diccionarios, como otros recursos lexicográficos que citaremos más adelante, ya dan cuenta -aunque no de forma homogénea- de los falsos amigos léxicos (San Vicente, 2008: 22, 223, 308). Dada la complejidad, a veces, es necesario integrar varias fuentes para completar la información. 


\subsection{DICCIONARIOS BILINGÜES}

Como premisa, debemos advertir que no todos los diccionarios bilingües de ámbito italoespañol recogen estos contenidos, no todos los falsos amigos figuran como tales en los diccionarios que tratan esta interferencia lingüística y no siempre se describen de manera eficaz (obligando al usuario a recurrir a otra fuente de consulta). A continuación, mostraremos algunos ejemplos, con el fin de reflejar el estado de la cuestión.

Tabla 1 Ejemplos de falsos amigos en los diccionarios Garzanti y Zanichelli

\begin{tabular}{|c|c|c|}
\hline & GARZANTI & ZANICHELLI \\
\hline $\begin{array}{l}\text { caballo de batalla } \\
\text { (español } \\
\text { peninsular: "punto } \\
\text { débil") }\end{array}$ & $\begin{array}{l}{[\ldots] \text { nodo critico, tasto dolente; }} \\
\text { cavallo di battaglia, pezzo forte }\end{array}$ & [...] cavallo di battaglia \\
\hline nómina & $\begin{array}{l}\text { 1. lista, elenco } \\
\text { 2. organico } \\
\text { 3. busta paga, foglio paga }[\ldots]\end{array}$ & $\begin{array}{l}{[\ldots] \text { FALSI AMICI: nómina non }} \\
\text { significa nomina }\end{array}$ \\
\hline novela & $\begin{array}{l}\text { 1. romanzo }[\ldots] \\
\text { 2. storiella }[\ldots] \\
\text { FALSO AMIGO. Da non } \\
\text { confondere con l'italiano } \\
\text { novella', che si traduce cuento. }\end{array}$ & $\begin{array}{l}{[\ldots] \text { FALSI AMICI: novela nel }} \\
\text { linguaggio comune non significa } \\
\text { novella. }\end{array}$ \\
\hline sueldo & $\begin{array}{l}\text { stipendio } \\
\text { FALSO AMIGO. Da non } \\
\text { confondere con l'italiano } \\
\text { 'soldo', che al singolare si traduce } \\
\text { moneda e al plurale dinero. }\end{array}$ & $\begin{array}{l}\text { [... }] \text { FALSI AMICI: sueldo non } \\
\text { significa soldo nel senso di denaro. }\end{array}$ \\
\hline
\end{tabular}

Entre las obras mejores y más utilizadas en ámbito italoespañol (Calvo Rigual, en prensa), hemos seleccionado el diccionario bilingüe de Arqués y Padoan (2012), publicado por Zanichelli y el diccionario dirigido por Paola di Cataldo (2009), editado por Garzanti. En ambas obras se señala la presencia de falsos amigos, anunciándolo desde la portada y el prólogo, aunque en verdad no se trate esta cuestión de forma exhaustiva, dado que no llegan a 100 voces. Por otra parte, en los dos diccionarios se adopta una orientación unidireccional, esto es, se aporta información útil exclusivamente para los usuarios italianos. Como podemos apreciar en los ejemplos presentados, la información recogida es breve, incluso imprecisa y, en todo caso, carente de combinatoria o ejemplos esclarecedores. En el caso de Zanichelli, la señalización de los falsos amigos figura solo en la parte español-italiano y está pensada para que el usuario realice una segunda búsqueda. Echamos en falta una mayor sistematicidad en la identificación del falso amigo en el conjunto del lemario y, con respecto a aquel que figura como tal en estos 
diccionarios, una cantidad de información suficiente, que no obligue al usuario a realizar nuevas búsquedas o recurrir a otras fuentes.

\subsection{OTROS RECURSOS LEXICOGRÁFICOS}

La información proporcionada por el diccionario bilingüe se integra con otras fuentes de consulta. En este caso, nos centraremos en las herramientas lingüísticas de uso lexicográfico en formato electrónico. Actualmente, existe un gran número de recursos lexicográficos que contribuyen al aprendizaje y la consolidación del conocimiento de diferentes lenguas, como metadiccionarios (Word Reference, Reverso o Lexicool), bases de datos terminológicas (como IATE: Interactive Terminology for Europe), corpus lingüísticos (Sketch Engine, Corpus dell' italiano contemporaneo), traductores automáticos (Google translate, DeepL, Apertium) o incluso buscadores (Google, GoGoDuck, Yahoo...) y, por supuesto, diccionarios multilingües.

PORTLEX es un buen ejemplo de diccionario multilingüe en línea, una obra lexicográfica centrada en la frase nominal, basada en la teoría valencial, en la que se analizan la estructura argumental y los roles semánticos de los sustantivos en diferentes lenguas: castellano, francés, alemán, gallego e italiano. Gracias a la información aportada en el interior del artículo, es fácil desambiguar el significado de los nombres mediante sus combinaciones sintáctico-semánticas, la información morfológica y los ejemplos que se aportan, procedentes de corpus. Se puede consultar como un diccionario monolingüe, bilingüe o multilingüe. Y la lengua vehicular es el español. Este es, además, un diccionario en línea, semicolaborativo, que se va actualizando gracias a la interacción con el usuario.

Como podemos apreciar en las imágenes, PORTLEX puede ser consultado como obra monolingüe o bilingüe (pudiendo seleccionar el par de lenguas entre los cinco idiomas que componen este diccionario de la frase nominal). Presenta información morfológica, semántica y sintáctica, centrando su atención de modo especial en la combinatoria (teoría valencial), que es ejemplificada a través de un corpus anotado.

Partiendo de PORTLEX y con la finalidad de proporcionar dos simuladores actanciales a este diccionario multilingüe que sigue creciendo, han nacido MultiGenera (Generación multilingüe de estructuras argumentales del sustantivo y automatización de extracción de datos sintáctico-semánticos. Proyecto financiado por las Ayudas Fundación BBVA a equipos de investigación científica en el área de Sociedad Digital y Humanidades Digitales 2017) y MultiComb (Generador multilingüe de estructuras argumentales del sustantivo con aplicación en la producción en lenguas extranjeras, financiado por FEDER/ Ministerio de Economía, Industria y Competitividad-Agencia Estatal de Investigación. FFI2017-82454-P), cuya finalidad es la optimización de recursos lexicográficos ya existentes como corpus y diccionarios. 
Contrato (Singular y Plural, Masculino) Castellano

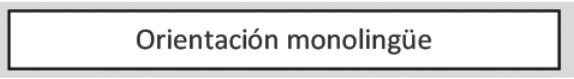

Sinónimos: Documento, Convenio

Definición:

Documento escrito en que queda reflejado un convenio.
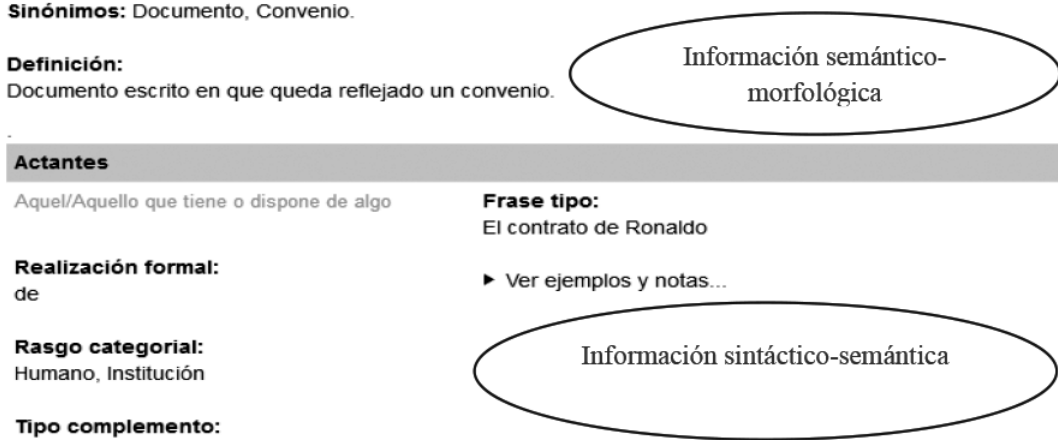

Tipo complemento:

Complemento sujeto

Frase tipo:

El contrato entre el productor y el distribuidor

- Ver ejemplos y notas..
Realización formal:

entre

Rasgo categorial:

Humano, Institución

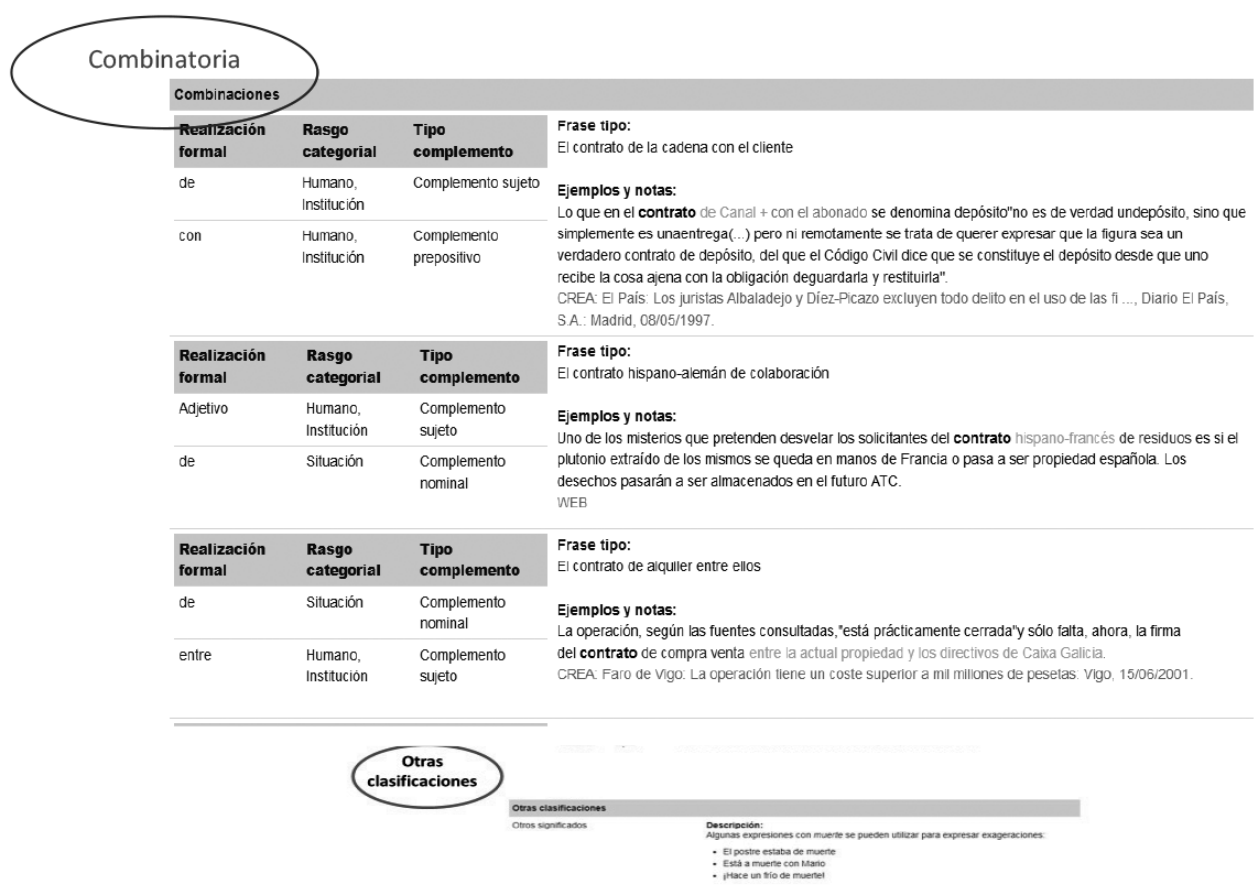

Figura 1 Segmento del artículo contrato (español) en PORTLEX, consulta monolingüe 


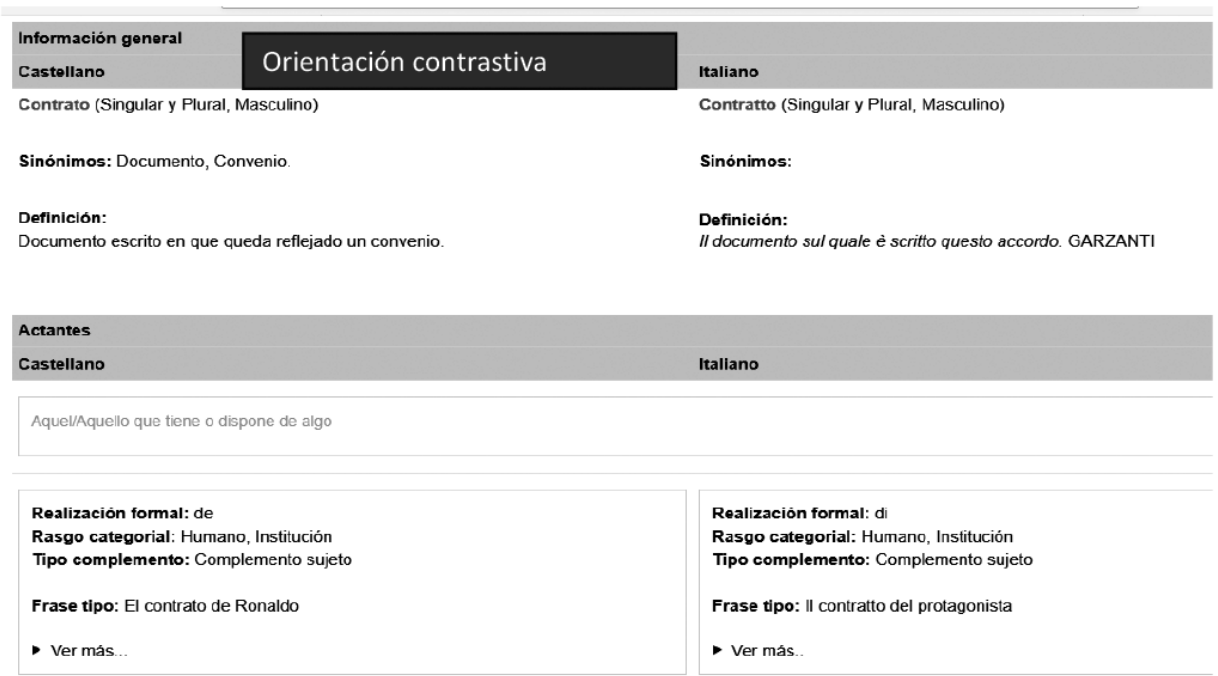

Figura 2 Segmento de contrato (español) y contratto (italiano) en PORTLEX, consulta bilingüe

Estos dos proyectos están orientados a la creación de aplicaciones lexicográficas, cuya finalidad es facilitar el aprendizaje y conocimiento de la lengua (Domínguez Vázquez, en prensa). MultiGenera permite la generación automática de frases nominales a partir de las estructuras argumentales y los roles semánticos propuestos en PORTLEX. Es un prototipo basado en diez sustantivos correspondientes a diferentes áreas de conocimiento (aumento, discusión, dolor, huida, pregunta, presencia, muerte, olor, sabor y texto). Se está desarrollando para tres idiomas, francés, alemán y español, pero en breve se ampliará al italiano, entre otras lenguas. Partiendo del etiquetaje semántico de estos sustantivos, combina herramientas propias -creadas ad hoc-con bases de datos ya existentes (como WordNet y Sketch Engine) para desarrollar un generador de frases nominales de manera automática (el sabor de los plátanos, el sabor frutal, el sabor a melón, el sabor a menta del helado...). MultiComb es también un prototipo, corresponde a la fase siguiente de la investigación: se fundamenta en las funciones léxicas de Mel'čuk (2013) y su objetivo es añadir el contexto a la frase nominal. De esta manera, se consigue generar automáticamente el marco oracional, añadiendo los adjetivos que acompañan al nombre, además de los verbos que rigen las frases nominales (me gusta mucho el fresco sabor a melón, el sabor a menta le repugnaba...). Ambas herramientas están en fase de elaboración y, en breve, serán de libre acceso. Además de nutrir de ejemplos al diccionario multilingüe de la frase nominal PORTLEX, serán de gran utilidad para tanto para la didáctica de lenguas como para la traducción. 


\section{CONCLUSIÓNES}

En la didáctica de las lenguas afines, cuantas más medidas preventivas se adopten para evitar una incorrecta asimilación de contenidos, mayor será el éxito en el proceso de aprendizaje. Hoy en día tenemos a nuestra disposición numerosos recursos lexicográficos, con diferentes orientaciones y finalidades: desde el diccionario bilingüe hasta las bases de datos lingüísticas o los metadiccionarios. $\mathrm{Si}$ recurrimos a la consulta combinada de varios recursos lexicográficos, se multiplican las posibilidades de obtener la información necesaria, desde el punto de vista semántico, cultural y pragmático.

En este contexto de interferencias lingüísticas y culturales, se vuelve imprescindible el poder contar con un entorno contrastivo sintáctico - semántico, con el objeto de poder prevenir las asimilaciones lingüísticas erróneas. Por esta razón, consideramos que las utilidades lingüísticas de MultiGenera y MultiComb, herramientas de procesamiento y generación automática que también abarcarán el italiano, constituyen un recurso lexicográfico imprescindible en la didáctica de la L2 y en el aprendizaje de una lengua afín.

\section{REFERENCIAS BIBLIOGRÁFICAS}

Álvarez Lugrís, A. (1997) Os falsos amigos da traducción: criterios de estudio e clasificación. Vigo: Universidade de Vigo.

Bailini, S. L. (2012) Interacción y desarrollo de la interlengua: e-tándem español-italiano. Tesis doctoral. Dir.: Vicente González Martín. Salamanca: Universidad de Salamanca.

Calvi, M. V. (1995) Didattica delle lingue affini: spagnolo e italiano. Milano: Guerini.

Calvo Rigual, C. (en prensa) La lexicografía italo-española: panorama actual y perspectivas de futuro. En D. Sánchez Palomino (ed.) Lexicografía iberorrománica. Madrid: Arco Libros.

Carlucci, L. y Díaz Ferrero, A. M. (2007) Falsas equivalencias en la traducción de lenguas afines: propuesta taxonómica. Sendebar: Revista de la Facultad de Traducción e Interpretación, 18: 159-190.

Domínguez Vázquez, M. J., Sanmarco Bande, M. T., Solla, M. y Valcárcel Riveiro, C. (en prensa) Die Einsetzung von neuartigen online Werkzeugen im Fremdsprachenunterricht: multilinguale automatische Generatoren des nominalen Kombinationspotentials. Aussiger Beiträge. Germanistische Schriftenreihe aus Forschung und Lehre. Acta universitatis purkynianae. Facultatis philosophicae studia germanica.

Gouws, R. H. (2017) La sociedad digital y los diccionarios. En M. J. Domínguez Vázquez y M. T. Sanmarco Bande (eds.) Lexicografía y didáctica (pp. 17-34). Nueva York: Peter Lang.

Mel'čuk, I. (2013) Semantics. From meaning to text. Amsterdam, Philadelphia: John Benjamins. Mello, L. y Satta, A. M. (1998) Falsi amici, veri nemici? Diccionario de similitudes engañosas entre el italiano y el español. México D.F.: Universidad Nacional Autónoma de México.

San Vicente Santiago, F. (2008) Textos fundamentales de la lexicografía italoespañola (1917-2007). Monza: Polimetrica.

Sanmarco Bande, M. T. (2018) Herramientas lexicográficas en el aula de Italiano L2. Quaderns d'Italià, 23: 117-130. 


\section{RECURSOS DE ANÁLISIS EMPÍRICO}

Apertium. Disponible en https://www.apertium.org/index.spa.html?dir=ita-spa\#translation [Consultado el 17 de junio de 2019].

Arqués Corominas, R. y Padoan, A (dir.), (2012) Il Grande dizionario di spagnolo. Dizionario spagnolo-italiano, italiano-spagnolo. Bolonia: Zanichelli.

Corpus dell'italiano contemporaneo. PAISÁ. Disponible en http://www.corpusitaliano.it/it/ index.html [Consultado el 17 de junio de 2019].

DeepL. Disponible en https://www.deepl.com/translator [Consultado el 17 de junio de 2019]. di Cataldo, P. (dir.), (2009) Il Grande dizionario spagnolo: spagnolo-italiano, italiano-spagnolo. Milano: Garzanti Linguistica.

GoGoDuck. Disponible en https://duckduckgo.com/ [Consultado el 17 de junio de 2019]. Google. Disponible en https://google.com/ [Consultado el 17 de junio de 2019].

Google translate. Disponible en https://translate.google.com/ [Consultado el 17 de junio de 2019].

IATE: Interactive Terminology for Europe. Disponible en https://iate.europa.eu/home [Consultado el 17 de junio de 2019].

Lexicool. Disponible en https://www.lexicool.com/ [Consultado el 17 de junio de 2019].

MultiComb. Disponible en http://portlex.usc.gal/multicomb [Consultado el 17 de junio de 2019].

MultiGenera. Disponible en http://portlex.usc.gal/multigenera [Consultado el 17 de junio de 2019].

PORTLEX. Disponible en http://portlex.usc.gal [Consultado el 17 de junio de 2019].

Reverso. Disponible en http://diccionario.reverso.net/[Consultado el 17 de junio de 2019].

Sketch Engine. Disponible en https://www.sketchengine.co.uk/ [Consultado el 17 de junio de 2019].

Word Reference. Disponible en http://www.wordreference.com/it/ [Consultado el 17 de junio de 2019].

Yahoo. Disponible en https:// it.yahoo.com/ [Consultado el 17 de junio de 2019].

\section{FALSE LEXICAL FRIENDS AND ELECTRONIC LEXICOGRAPHIC RESOURCES IN THE SPANISH-ITALIAN CONTEXT}

Abstract. The contrastive lexicography dealing with the pair of Italian-Spanish
languages has evolved considerably over the last decade, but there are still areas
where the information provided in dictionaries is still insufficient. The same
often happens with false friends (Spanish terms such as defraudar, larva, tesitura
or Italian, like indiano, salire, versare), due to the fallacious proximity between the
two languages. This sense of closeness often prevents the recognition of existing
differences, reinforcing the weight of cultural stereotypes and thus invisibilizing
the distinctive elements of each of the two languages and of the two cultures.
The purpose of our paper is to show how this linguistic-cultural divergence is
reflected in the most widely used Italian-Spanish bilingual dictionaries and,
to this end, we will focus on the treatment of false friends present in the most
recent dictionaries, those published by Garzanti and Zanichelli. After showing 
how this information is reflected in the dictionaries in use, we offer a series of electronic resources (dictionaries, databases, applications... some in the process of elaboration, such as MultiGenera and MultiComb), focusing on how they can complement contents about false friends. Our final aim is to offer an overview of the lexicographic resources available for completing the definitions of false friends, contributing in this way to metalexicographic criticism.

Key words: false lexical friends, Italian-Spanish, bilingual lexicography, electronic lexicographical resources, MultiGenera, MultiComb

Rosa Martín Gascueña (Dra., Prof.a contratada doctora) imparte docencia en la Universidad Complutense de Madrid. Sus campos de investigación son la lingüística aplicada, la semántica léxica y el análisis del discurso. Correo electrónico: rosamartingascuena@filol.ucm.es

María Teresa Sanmarco Bande (Dra., Prof. ${ }^{a}$ titular) trabaja actualmente en la Universidad de Santiago de Compostela. Sus intereses académicos incluyen la lexicografía, la lexicología y la lingüística contrastiva en ámbito italoespañol. Correo electrónico: mt.sanmarco@usc.es 